\title{
TRATAMENTO DOS ANEURISMAS DO SISTEMA DA ARTÉRIA CEREBRAL ANTERIOR
}

\author{
Mário S. Cademartori * \\ Nelson Pires Ferreira* \\ Felipe WAINeR ** \\ ROBERTO WAGNER **
}

Entre junho de 1960 e junho de 1972 foram atendidos, no Instituto de Neurocirurgia de Porto Alegre, 206 casos de aneurismas intracranianos, dos quais $63(29 \%)$ eram do sistema da artéria cerebral anterior. A idade e o sexo dos pacientes é apresentada no quadro 1. A localização dos aneurismas é mostrada no gráfico 1 .

\begin{tabular}{|c|c|c|c|c|c|}
\hline \multirow{2}{*}{\multicolumn{2}{|c|}{$\begin{array}{l}\text { Idade } \\
\text { (anos) }\end{array}$}} & \multicolumn{2}{|c|}{ Sexo } & \multirow{2}{*}{\multicolumn{2}{|c|}{ Totais }} \\
\hline & & \multirow{2}{*}{$\mathbf{M}$} & $F$ & & \\
\hline & 20 & & 1 & 1 & $(1,58 \%)$ \\
\hline & 30 & 4 & 2 & 6 & $(9,52 \%)$ \\
\hline & 40 & 9 & 9 & 18 & $(28,60 \%)$ \\
\hline & 50 & 14 & 8 & 22 & $(34,92 \%)$ \\
\hline & 60 & 8 & 4 & 12 & $(19,04 \%)$ \\
\hline & 70 & & 3 & 3 & $(4,76 \%)$ \\
\hline \multicolumn{2}{|c|}{ Ignorada } & & 1 & 1 & $(1,58 \%)$ \\
\hline
\end{tabular}

A maneira de tratar estes aneurismas passou por fases diferentes.

Periodo de 1960 a $196 \%$ - Neste período os resultados publicados, na literatura e relativos ao tratamento cirúrgico eram controvertidos 5, 7, 9, 10, 11, 15 . A comparação dos resultados entre o tratamento conservador e o ataque direto era favorável ao primeiro, sendo ambos pouco animadores. Tais fatos dividiam as opiniões, inclusive em nosso meio, resultando em prolongada hospitalização de pacientes, ocorrendo ressangramentos e elevada mortalidade préoperatória $(36,8 \%)$. A conduta não intervencionista, defendida principalmente por Mekissock e col. ${ }^{9}$, era predominante. Fm outros serviços eram preconizados novos critérios de investigação e tratamento ${ }^{12}$. Diversos procedimentos

Trabalho do Instituto de Neurocirurgia de Porto Alegre, apresentado no IX Congresso Brasileiro de Neurocirurgia (Rio de Janeiro GB, 1972): * Neurocirurgiões; ** Médicos residentes. 


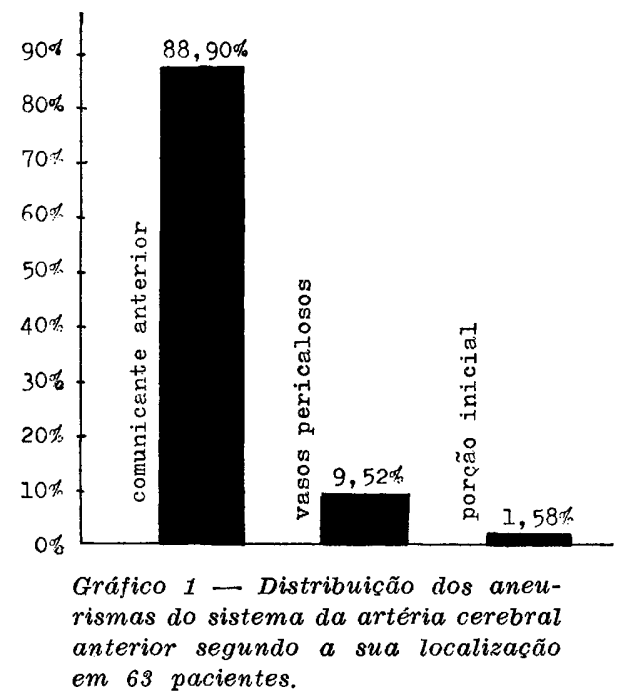

cirúrgicos foram recomendados para tratar tais aneurismas: ligadura da carótida, da cerebral anterior dominante, clipagem, envolvimento, mudança de carga elétrica e trombose, abordagem através de trepanação uni ou bifrontal, com e sem hipotermia $2,3,8,14,16$. Em nosso meio quase tudo foi tentado e, de 19 pacientes atendidos, oito foram submetidos a intervenção, com mortalidade elevada $(50 \%)$. Tomavamos consciência da necessidade de melhorar o instrumental, os clips e os aspiradores, assim como de padronizar a abordagem. Já era marcante a relação entre a situação clínica e o resultado operatório.

Periodo entre $196 \%$ e 1970 - Enquanto que no periodo anterior de 19 pacientes, apenas oito foram operados, neste, de 22 pacientes, 14 foram submetidos à cirurgia, com mortalidade de $21 \%$. Nossos resultados já eram mais encorajadores. Este período foi claramente definido, pela valorização dos "bons" e "maus" riscos cirúrgicos ${ }^{15}$. Foi utilizada a drenagem espinal continua sistematicamente, a abordagem sub-frontal unilateral sem lobectomia $^{1,4}$ substituiu a bifrontal ${ }^{12}$, foi melhorada a produção de hipotermia $\mathrm{e}$ utilizada a hipotensão arterial durante a dissecção dos aneurismas. Os cirurgiões, já mais experimentados, dispunham de uma via de acesso padronizada ${ }^{1}$, de clips e aspiradores apropriados. Dois métodos de tratar o aneurisma passaram a ser rotina - clipagem e revestimento - na dependência da situação, volume e forma dos aneurismas.

Periodo de 1970 a 1972 - Neste período foram operados 19 de 22 pacientes, com mortalidade de $15,7 \%$. A redução da mortalidade em relação aos periodos anteriores foi apreciável e definitivamente influenciada pela maior experiência e seleção de casos. 
A mortalidade em época sucessivas pode ser vista no gráfico 2 .

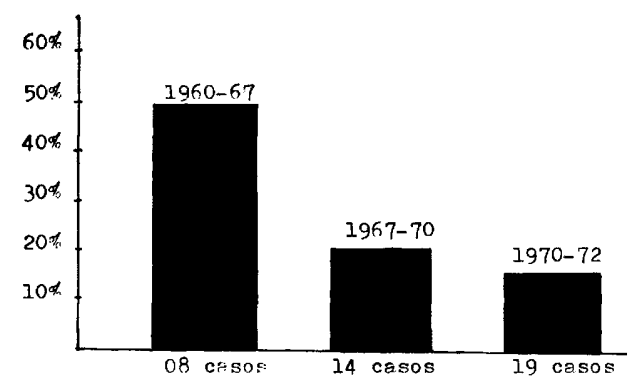

Gráfico 2 - Diminuiç̃o da mortalidade operatória nos três periodos considerados em virtude da selecão de pacientes, da uniformidade de abordagem cirúrgica e da maneira de tratar os aneurismas.

Arteriografia - Com base nos resultados apresentados em trabalhos publicados entre 1966 e 1970, a arteriografia foi realizada geralmente na primeira semana e, nos últimos dois anos, efetuada em média no $12 .^{\circ}$ dia. A revisão de alguns casos de pacientes que faleceram antes da intervenção cirúrgica mostrou que a situação clínica é de transcendental importância para escolher o dia em que este exame deve ser feito. $O$ critério generalizado de efetuar angiografia logo após a verificação de hemorragia, foi de pouco valor no nosso pequeno número de casos. Exceto no que diz respeito à suspeita de ocorrência de hematomas volumosos, esta conduta tem sido contraindicada nos casos de pacientes sonolentos e bradipsíquicos, com crises hipertensivas e distúrbios vegetativos. O quadro 2 torna evidente que o exame foi inoportunamente indicado em alguns casos. A presença de espasmos arteriais, às vezes insuspeitados clinicamente, tem sido motivo para postegar o exame, medida que acreditamos tenha salvo alguns pacientes. Na suspeita de aneurisma, a arteriografia é feita exclusivamente em casos enquadráveis em grau I ou estabilizados. Salientamos o registro de 18 casos de trifurcação da carótida, por agenesia ou hipoplasia de uma das cerebrais anteriores. Este fato adicionado à morfologia e situação dos aneurismas serve de orientação para o tratamento a ser dado ao aneurisma e a necessidade de poupar a artéria cerebral anterior dominante.

Tratamento cirúrgico - Não existindo outras causas que contra-indiquem a cirurgia nossa conduta é intervencionista. Operamos pacientes assintomáti$\cos$ ou aqueles cujos déficits tenham atingido estabilidade. Nos últimos dois anos o período médio entre a hemorragia e o ato cirúrgico foi de 21 dias (Quadro 3). Para a intervenção nossos pacientes são colocados assentados ou em decúbito dorsal, com a cabeça em boa posição para facilitar a drenagem venosa. Utilizamos sistematicamente drenagem espinal continua, com 
retirada de 40 a $80 \mathrm{ml}$ de líquido cefalorraqueano, sem reposição no final da operação. A via de acesso utilizada por um dos autores a partir de $1967^{1}$, e atualmente utilizada como rotina, é feita mediante trepanação triangular, baixa, indiferente ao volume do seio frontal, sendo feitos três furos: um mediano (nasal); outro lateral, o mais afastado que for possivel do primeiro e junto ao rebordo orbitário; outro a $2 \mathrm{~cm}$ da sutura mediana e aproximadamente a $12 \mathrm{~cm}$ do rebordo orbitário. Avalia-se o colapso frontal continuando-se ou não, a drenagem de líquido cefalorraqueano. Por meio de espátulas, estreitas e maleáveis, pratica-se abordagem subfrontal. As vezes é necessário coagular e seccionar algumas veias cortico-durais e seccionar o nervo olfativo, afim de que o lobo frontal possa ser afastado. Aborda-se a apófise clinóide anterior, disseca-se a aracnóide do nervo óptico e da porção inicial do quiasma. Pequenos sangramentos venosos ou arteriais podem então ocorrer, sendo controlados por tamponamento temporário com pastas de algodão. Disseca-se a aracnóide que recobre a carótida que é exposta até a bifurcação. A artéria cerebral anterior é exposta até sua porção ascendente. O aneurisma já é, então, visível e pequenas bridas de aracnóide podem estar aderidas ao seu fundo quando projetado anterior ou inferiormente sobre a região quiasmática. Coloca-se, então, uma espátula estreita suportada por um prendedor fixo ao osso e pratica-se a dissecção e exposição final do aneurisma. Para expo-lo adequadamente são utilizadas lentes de aumento e dissectores delgados e estreitos. A dissecção é facilitada pelo emprego de aspirador de $2 \mathrm{~mm}$ de diâmetro e ponta romboide, cuja força de sucção é reduzida, quando se aborda o saco aneurismático, para evitar retirada de pequenos coágulos que possam estar tamponando fissuras recentes. As manobras de exposição devem ser delicadas, perseverantes e pacientes para evitar, ao máximo, causas mecânicas que possam originar espasmos vasculares. Aneurismas de forma irregular, de grande tamanho com projeção superior ou posterior, para serem expostos, necessitam de pequenas lobectomias do giro reto. A exposição completa da ectasia permite identificar sua implantação, sua forma e relações anatômicas com as artérias pericalosas, mesodiencefálicas e ramúsculos hipofisários e ópticos, os quais devem ser interrompidos. Quando os aneurismas são alongados e de projeção anterior ou inferior é conveniente e possível dissecar somente o colo, sem manipular o fundo, ponto fragil e capaz de romper. Se for necessária uma exposição mais ampla é preciso seccionar bridas de aracnoide entre o fundo da malformação e o quiasma e região selar. Uma vez exposto o aneurisma poderá ser clipado. A aplicação correta do clipe deve evitar a interrupção de alguma das artérias da vizinhança acima referidas. Caso isto tenha ocorrido, o clipe deverá ser retirado e reaplicado. $\mathrm{Na}$ imposibilidade de clipar, revestimos o aneurisma com tela de nylon, procedimento utilizado em nosso meio desde 1966.

Existindo hematomas estas coleções sangüineas devem ser retiradas por via subfrontal, antes da abordagem ao aneurisma. Concluida a intervenção cirúrgica testa-se a hemostasia, fecha-se a dura-mater, recobre-se o seio frontal (caso tenha sido aberto) com gelfoam, impregnado de antibiótico, fixa-se o osso, sutura-se a galia e a pele. 


\begin{tabular}{ccccc} 
Grau & $\begin{array}{c}\text { N.o de } \\
\text { casos }\end{array}$ & $\begin{array}{c}\text { Intervalo entre a } \\
\text { hemorragia e exame } \\
\text { (em dias) }\end{array}$ & $\begin{array}{c}\text { Incidência } \\
\text { de } \\
\text { espasmos }\end{array}$ & $\begin{array}{c}\text { Intervalo } \\
\text { decorrido até } \\
\text { o óbito } \\
\text { (em dias) }\end{array}$ \\
III & 1 & 16 & não & 6 \\
III & 1 & 8 & não & 3 \\
III & 1 & 1 & não & 23 \\
III & 1 & 6 & não & 0 \\
IV & 1 & 2 & sim & 6 \\
IV & 1 & 5 & sim & \\
\hline
\end{tabular}

Quadro 2 - Pacientes em condicões clínicas inadequadas e submetidos inoportunamente à angiografia.

\begin{tabular}{cccc}
\hline Períodos & $\begin{array}{c}\text { Intervalo entre } \\
\text { hemorragia } \\
\text { e arteriografia } \\
\text { (média em dias) }\end{array}$ & $\begin{array}{c}\text { Intervalo entre } \\
\text { hemorragia } \\
\text { e cirurgia } \\
\text { (média em dias) }\end{array}$ & $\begin{array}{c}\text { Mortalidade } \\
\text { cirúrgica }\end{array}$ \\
\hline $1960-1967$ & 7 & 30 & $50 \%$ \\
$1967-1970$ & antes do 7.0 & 7 & $21 \%$ \\
$1970-1972$ & 12 & 21 & $16 \%$ \\
\hline
\end{tabular}

Quadro 3 - Períodos de tempo decorridos entre a hemorragia e investigacão arteriográfica e entre a hemorragia e o ato cirúrgico na metódica empregada durante o período de 1960 até 1972.

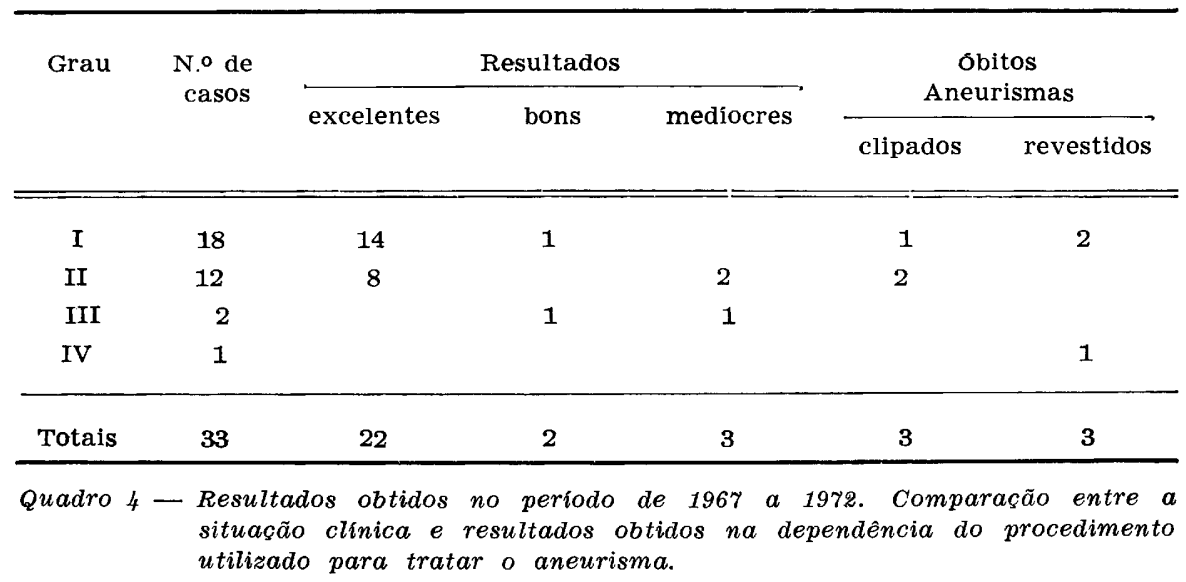


Os pacientes são mantidos, no pós-operatório imediato, com o tronco e a cabeça moderadamente levantados. O equilíbrio hidroeletrolítico é verificado de rotina. Em nenhum de nossos casos ocorreram fístulas de líquido cefalorraqueano.

De 1967 até 1972 foram operados 33 pacientes (Quadro 4), sendo que os aneurismas foram revestidos em 14 casos e clipados nos 19 restantes. Faleceram 3 pacientes do primeiro grupo - um no grau 4 e dois no grau 1 um por ressangramento e, outro, por rotura transcirúrgica. Nos últimos dois anos, de 19 pacientes operados, faleceram três, cujos aneurismas foram clipados: um por tentativa de clipar pequeno aneurisma que rompeu, sendo clipada uma das pericalosas (grau 1); um (grau 3) indevidamente encaminhado à cirurgia (aneurismas múltiplos e trombose antiga de uma das carótidas); outro, de grau 2, por causa indeterminada no $23 .^{\circ}$ dia após a intervenção cirúrgica. Entre os pacientes nos quais foi feito revestimento não ocorreu óbito.

Continuamos pensando e sabendo que muito deve ser melhorado, acreditamos que o advento da microcirurgia trará apreciável contribuição ao tratamento cirúrgico destes aneurismas, mas enquanto não a pudermos utilizar como rotina a via simples de acesso que utilizamos é um fator a favor do paciente e do cirurgião.

\section{R E S U M O}

Os autores analisam a evolução do tratamento cirúrgico de aneurismas do sistema da artéria cerebral anterior no Instituto de Neurocirurgia de Porto Alegre, de 1960 a junho de 1972. Foram operados 63 pacientes, dos quais 41 por ataque direto. Consideram que o estudo angiográfico só deve ser feito em pacientes em condições clínicas satisfatórias ou estabilizadas. Descrevem pormenorisadamente a técnica cirúrgica preconisada em 1967 por um dos autores e o método de tratar tais aneurismas desde então. Apresentam quadros demonstrativos dos resultados obtidos em épocas sucessivas e os resultados globais alcançados. Acreditam que as técnicas atuais de tratamento serão favorecidas pela microcirurgia, mas uma via de acesso simples será sempre um fator favorável.

\section{S U M M A R Y}

\section{Surgical treatment of the anterior cerebral system aneurysms}

Sixty three cases of anterior cerebral artery aneurysms observed in the period of 1960-1972 were reviewed on basis of their surgical approaches. The authors claim the necessity to perform intracranial vessels arteriography only in alert patients, except when haematoma is to be suspected. The surgical procedure consists in a frontal triangular craniotomy, followed by a subfrontal approach to exposing the aneuysm. Superior posterior implan- 
tantion of the aneurysmal sac may require partial ablation of the girus retus. In the same way continuous cerebrospinal fluid drainage facilitates the approach, as the arterial hypotension enables a safer neck dissection. Only clippsing and wrapping techniques are now employed. The rate mortality was $50 \%$ between 1960 and $1967,18 \%$ in the period $1967-72$ and $16 \%$ in 19 cases operated in the last two years.

\section{REFEREN CIA S}

1. CADEMARTORI, M. S. - Considerações clinicas e cirúrgicas sobre 115 casos de aneurismas intracranianos. Neurobiologia (Recife) 36:231, 1970.

2. COOK, A. W.; DONALD, M.; DOOLEY, D. M. \& BROWDER, E. J. - Anterior communicating aneurysms. Treatment by ligation of an anterior cerebral artery. J. Neurosurg. 23:371, 1965 .

3. DRAKE, C. G.; BARR, H. W. K.; COLES, J. C. \& GERGELY, N. F. - The use of extracorporeal circulation and profound hypothermia in the treatment of ruptured intracranial aneurysms. J. Neurosurg. 21:575, 1964.

4. FRENCH, L. A.; ZARLING, M. E. \& SCHULTZ, E. A. - Management of aneurysms of the anterior communicating artery. J. Neurosurg. 19:870, 1962.

5. FRENCH, L. A.; SHELHY, N. C.; STORY, J. L. \& SCHULTZ, E. - Aneurysms of the anterior communicating artery. J. Neurosurg. 24:1057, 1966.

6. KEMPE, L. C. \& VANDER ARK G. D. - Classification of anterior communicating aneurysms as a basis of surgical approach. J. Neurosurg. 33:300, 1970.

7. KIRGIS, H. D.; FISHER, W. L.; LIEWELLYN, R. C. \& Mc PEEBLES, C. E. - Aneurysms of the anterior communicating artery and gross anomalies of the circle of Willis. J. Neurosurg. 25:73, 1966.

8. MASPES, P. E. \& MARINI, G. - Results of direct surgical treatment with special reference of the use of moderate hypothermia and circulatory arrest. J. Neurosurg. 21:284, 1964.

9. MEKISSOCK, W.; PAINE, K. W. E. \& WALSH, L. S. - An analysis of the results of treatment of ruptured intracranial aneurysms; report of 772 consecutive cases. J. Neurosurg. 17:762, 1960.

10. MOUNT, L. A. - Results of treatment of intracranial aneurysms using silverstone clamp. J. Neurosurg. 16:611, 1959.

11. NORLEN, G. \& BARNAUM, A. S. - Surgical treatment of aneurysms of the anterior communicating artery. J. Neurosurg. 10:634, 1953.

12. POOL, J. L. - Timing and techniques in the intracranial surgery. J. Neurosurg. 19:378, 1962.

13. POOL, J. L. - Bifrontal craniotomy for anterior communicating artery aneurysms. J. Neurosurg. 36:212, 1972 .

14. SAHS, A. L. - Hypotension and hypothermia in the treatment of intracranial aneurysms. J. Neurosurg. 25:593, 1966.

15. SKULTETY, F. M. \& NISHIOKA, H. - The result of intracranial surgery in the treatment of aneurysms. J. Neurosurg. 25:683, 1966.

16. SUGAR, O. \& TSUCHIYA, G. - Plastic coating of intracranial aneurysms with EDH-adesive. J. Neurosurg. 21:114, 1964.

Instituto de Neurocirurgia - Hospital São Francisco - 90000 Porto Alegre, RS - Brasil. 